\title{
La traduction-fiction chez Nicole Brossard
}

Nicole Brossard: Fictionalising the Translation Process

\section{Angelo Vannini}

\section{(2) OpenEdition}

Journals

Édition électronique

URL : http://journals.openedition.org/recherchestravaux/1728

DOI : 10.4000/recherchestravaux. 1728

ISSN : 1969-6434

Éditeur

UGA Éditions/Université Grenoble Alpes

Édition imprimée

ISBN : 978-2-37747-165-2

ISSN : 0151-1874

Référence électronique

Angelo Vannini, « La traduction-fiction chez Nicole Brossard », Recherches \& Travaux [En ligne], 95

2019, mis en ligne le 05 décembre 2019, consulté le 08 septembre 2020. URL : http://

journals.openedition.org/recherchestravaux/1728; DOI : https://doi.org/10.4000/recherchestravaux. 1728

Ce document a été généré automatiquement le 8 septembre 2020

(c) Recherches \& Travaux 


\title{
La traduction-fiction chez Nicole Brossard
}

\author{
Nicole Brossard: Fictionalising the Translation Process
}

Angelo Vannini

1 En 1987, la poète québécoise Nicole Brossard publie un roman tout à fait singulier, dont le titre parle déjà un langage à la fois familier et inédit : Le Désert mauve ${ }^{1}$. Parle-t-il vraiment un langage, ou bien en parle-t-il plusieurs? Est-ce un violet mauve, un violet français, ou bien mauve, c'est-à-dire anglais ? Est-ce qu'il y aurait une différence, est-ce qu'un désert changerait de couleur, selon qu'on le dit en anglais ou en français?

2 C'est un titre qui parle abondamment, Le Désert mauve; il parle plusieurs langues, plusieurs couleurs et plusieurs choses. Car Le Désert mauve est aussi, avec un tout petit déplacement, le désir Maude... le désir à Maude. Voici l'histoire du roman : une femme, Maude Laures, trouve à Montréal un petit livre dans une librairie de livres usagés, écrit en langue étrangère par une inconnue nommée Laure Angstelle. Maude l'achète, le lit et est subjuguée par celui-ci à tel point qu'elle décide de le traduire en français. Le roman de Nicole Brossard est composé de trois parties : le récit original par l'auteure fictive Laure Angstelle, intitulé Le Désert mauve; un ensemble composite de réflexions, notes, scènes et descriptions, rédigé en partie par une voix extra-diégétique et en partie par la traductrice fictive Maude Laures, incluant aussi un dossier photographique; la traduction elle-même, intitulée Mauve, l'horizon. Or, ce qui fait le grand intérêt du livre, c'est non seulement que nous avons affaire à un roman qui fait de la traduction son thème principal et son enjeu essentiel, mais aussi le fait que tout dans ce roman est en français, y compris le récit original attribué dans la fiction à Laure Angstelle. C'est-à-dire que ce texte nous donne à lire une traduction du français au français. Qu'est-ce que c'est que cela, une traduction d'une langue à la même langue ?

On pourrait facilement penser de prime abord qu'il s'agit d'un divertissement tout en brio, une sorte d'épreuve de virtuosité de la part de l'auteure. Certes, une composante ludique est présente dans ce roman, comme en général dans l'écriture de Nicole Brossard ; mais nous nous méprendrions pour de bon si nous nous en tenions là. L'enjeu de cette expérience qu'est Le Désert mauve - une expérience à la fois d'écriture et de 
lecture - est au contraire profondément sérieux. Ce que je me propose de faire ici, c'est bien d'en dégager le "sérieux », autant que faire se peut dans les limites de quelques pages. Il y a à cet égard un certain nombre de questions que nous devrions nous poser. Une traduction du français au français est-elle une traduction? Si oui, sur quoi pourrait-elle porter? En d'autres termes, qu'est-ce que l'auteure aurait pu traduire d'une langue à la même langue ? Et surtout, qu'arrive-t-il à la langue par ce procédé ? Et qu'arrive-t-il à la traduction?

\section{La langue}

4 Tout d'abord la langue. Qu'arrive-t-il donc à la langue française ? Il y arrive l'anglais, puisque Le Désert mauve n'est pas monolingue, en dépit des apparences. Nous y trouvons en effet, dans le récit «original » de Laure Angstelle, que nous lisons en français mais qui dans l'univers fictionnel est pensé en langue étrangère, nous y trouvons çà et là des mots mis en relief, typographiquement, par les italiques. Voici un inventaire choisi, non exhaustif : « un jour, je serais fast so fast, sharp so sharp, un jour j'aurais devant la nécessité de l'aube tout oublié de la civilisation des hommes ${ }^{2} »$; ou bien dans la même page, un peu plus bas: "l'humanité était fragile parce qu'elle ne soupçonnait pas l'existence de l'Arizona. So fragile. J'avais quinze ans et je désirais que tout soit comme en la fragilité de mon corps ». Encore : «Une fin d'après-midi de mai, alors que j'avais quitté la route pour examiner de plus près un vieux saguaro à la silhouette mi-blessée, mi-agonique et que je chantais comme à l'accoutumée - fever, fever, forever m'en aller -, je sentais la peur pénible ${ }^{3}$ "; "Light me pour que le désert s'abîme en nous et que renaissent les ultrasons de notre enfance. Light me because I might un jour ${ }^{4}{ }^{\prime}$.

Que viennent faire ici ces mots en italique? Et surtout, ce sont des mots en quelle langue? Le français étant déjà, dans la fiction, une autre langue, l'anglais de ces mots acquiert un statut troublé, et surtout troublant. Ce sont des mots qui arrivent par effraction, qui fissurent le corps virtuel de la langue et qui, par le seul fait d'être là, démasquent la fiction, dénoncent la langue adaptée - le français - comme langue nonmimétique. C'est-à-dire qu'ils dénoncent la totalité du français, la qualité totalisante ou, si l'on veut, totalitaire de la langue - de toute langue, celle-ci comme toute autre. Et ce sont surtout des mots qui, de cette langue " totale $^{5}$ ", en redessinent le contour. Quel contour? Non pas, bien sûr, le contour extérieur de la langue - celui qui varie selon qu'on parle de la marge, de la périphérie ou du centre, comme l'explique Nicole Brossard dans un très bel essai consacré à la traduction ${ }^{6}$. Je parle ici de son contour intérieur, c'est-à-dire d'une profondeur qui devient si intime qu'elle paraît, ou est, autre. Aucune langue n'est pure : nous parlons anglais en français, mais aussi italien, allemand, latin, arabe... Dans le roman de Nicole Brossard, l'auteure, elle, s'appelle Angstelle. Est-ce du français, de l'anglais ou de l'allemand ? Peut-être néerlandais ?

Encore un autre passage du texte : «Grazie ${ }^{7}$ se passionna, moqueuse et curieuse, pour mon tatouage sur l'épaule gauche, en suivit le contour avec ses doigts doux, dit qu'un jour elle se ferait dessiner une licorne sur une fesse. Puis, nous avons préparé des sand witches comme dans notre enfance et j'ai bu deux litres d'eau ${ }^{8} »$. Du mot français sandwich, Nicole Brossard en fait éclater ici la substance: son origine anglaise, son altérité, devient propulsive jusqu'au point où le sandwich explose en sand witches, en... sable sorcières. Et nous voilà transportés, par alchimie langagière, d'une spécialité culinaire (masculine) que l'on mange souvent en vitesse, à un foisonnement de 
sorcières sablonneuses, bien sûr féminines et joyeusement préparées «comme dans notre enfance ".

7 En lisant Le Désert mauve de Nicole Brossard on ne sait plus quelle langue, entre l'anglais et le français, est la langue la plus proche. On pourrait se demander quelle est la langue la plus originaire, dans un sens génétique. On pourrait en effet penser que l'anglais n'opère ici que par intromission, qu'il vient seulement assaisonner un mets originairement francophone. On pourrait se dire par exemple que le rapprochement qui a lieu dans le texte entre la narratrice Mélanie et la nuit, ne peut exister qu'en français. Nous y lisons en effet : "Ma mère m'avait parlé d'un voyage à Dante's View, m'avait raconté promenade et point de vue, le plus saisissant à Dante's View, les plus beaux à Badwater et à Artist Drive. Puis elle avait ajouté : "Mélanie, mais la nuit" " ". Est-ce que ce passage serait pensable dans une autre langue? On est tenté de répondre que non, qu'à l'origine il y a le français, et le français seulement. Et pourtant. Voici un autre passage, tiré du dernier chapitre du récit de Laure Angstelle : «Que s'est-il passé ? C'était pourtant un homme de génie. Of course Mélanie is night teen ${ }^{10} »$. Night teen, au lieu de nineteen. Voilà que Mélanie est en anglais aussi une fille de la nuit.

8 Si l'on ne peut pas dire quelle langue est la plus originaire, c'est que Nicole Brossard met en scène dans Le Désert mauve une double altérité fictive. Et il s'agit d'une fiction qui permet aussi de saisir quelque chose du mystère de l'altérité, c'est-à-dire la présence, dans ce qui est "autre », d'un certain noyau de ressemblance : l'altérité ne peut jamais être " pure ", totalement différente, car il serait impossible, dans ce cas, de la reconnaitre comme autre. Une double altérité donc : l'altérité fictive du soi, avec l'une et l'autre Laure(s), l'auteure et la traductrice, dont la différence et la proximité sont soulignées par la quasi-identité du nom. Et c'est une identité en déplacement, celle qui glisse de Laure à Laures comme du prénom au nom, et du singulier au pluriel. Outre l'altérité fictive du sujet, il y a bien sûr celle de la langue. Me traduire dans ma propre langue, comme si ma langue était une autre langue; mais sur ce «comme si » il faudra réfléchir, car il s'agit au fond d'une fiction qui ne feint pas grand-chose, puisqu'il est au moins un sens par lequel je puis dire que ma langue est une autre langue. Au moins un sens, mais probablement plusieurs. Il y a bien sûr le sens dont Jacques Derrida a tenté de rendre compte ${ }^{11}$; mais il y a aussi un autre sens, dont témoigne toute l'œuvre de Nicole Brossard.

\section{La traductrice}

9 Je ne dis pas une chose nouvelle en affirmant que la plupart des approches de la traduction conçoivent celle-ci comme un "transfert de sens", ayant pour corollaire qu'une bonne traduction ne doit jamais paraitre comme une traduction, mais plutôt sembler être née dans une prétendue langue " cible "; par conséquent le traducteur ne doit pas laisser sa marque, il doit s'effacer autant que possible. C'est ce qu'Antoine Berman appelait l'« auto-oblitération de la traduction et du traducteur ${ }^{12}$ ».

Or, à l'intérieur du roman de Nicole Brossard, dans la partie qui sépare le récit de Laure Angstelle et la traduction, il y a une sous-section qui, rédigée par la traductrice, est consacrée aux personnages du récit original. Parmi ces personnages figure aussi la traductrice, évoquée par un autoportrait. Ceci n'est pas sans signification. La traductrice est présente, dans ce roman, à chaque pas ; je serais tenté de dire que même dans la première partie, là où nous lisons le texte "original » de Laure Angstelle, la 
traductrice Maude Laures est présente sous le mode de l'imminence, de l'anticipation ou de la prédestination ${ }^{13}$. En effet, Le Désert mauve est un roman non seulement sur la traduction, mais aussi, et peut-être plutôt, sur la traductrice. Fascinée par ce récit trouvé par hasard, Maude Laures décide de le traduire. Qu'est-ce que cela veut dire, qu'est-ce qu'une telle décision? Où prend-elle origine?

On pourrait penser - et à juste titre, je crois - que le roman de Nicole Brossard, ou du moins une bonne partie de ce roman, n'est en fait qu'une réponse à cette question, une sorte de généalogie non pas de la traduction, mais de la décision de traduire, ou mieux encore, du désir qui mènera à cette décision : un désir qui, pour s'assouvir, exigera de la part de Maude cet acte extrême de lecture que l'on nomme traduction. Par ailleurs, le désir est l'un des thèmes principaux du roman. Dès que Maude Laures trouve le livre, tout son être s'y enfonce, éprouvant un désir lancinant. Pourquoi? Voici la question que tout lecteur ou lectrice est amené à se poser. En tout cas, il s'agit d'un désir qui ne reste pas confiné à l'univers fictionnel; au contraire, il est concrètement et à tout moment palpable par les lecteurs, car il anime et est animé par l'écriture de Nicole Brossard. C'est un désir que nous voyons circuler, même quand il n'est pas nommé, à chaque page du livre.

Non seulement ce roman met en scène la traductrice, mais aussi son parcours dans la traduction. Au début, Maude n'est qu'une " présence minimale, un jalon entre ce livre et son devenir dans une langue autre ${ }^{14} »$; malgré sa bonne volonté, la traduction du récit commence à lui poser problème, car elle y découvre des données sensorielles qu'elle n'arrive pas à faire siennes. Maude essaye alors d'entrer "par le détail » dans l'univers de la narratrice, mais les difficultés qu'elle rencontre la refoulent dans son propre monde. Son désir fou se traduit ici en une peur panique de se substituer à l'auteure du livre, de ce livre qui sans préavis a sapé son équilibre. Ensuite, Maude entrevoit la possibilité d'opérer quelques transformations; elle comprend qu'il faut trouver la faille, le petit endroit où le sens appelle quelques audaces. Mais elle vit encore seulement dans l'intention de faire passer, par des allers-retours répétés, jusqu'au moment où quelque chose se passe. Jusqu'à ce que soudain elle comprenne que l'expression n'est qu'une "proposition capable de faire pencher la balance ${ }^{15}$ ». Il ne s'agit plus maintenant de faire passer, mais de faire valoir le passage dans sa langue, d'accélérer, écrit Brossard, le sentiment : «Alors elle cherchait l'envers des mots, avec un peu d'affolement, la doublure, lorsque la scène lui paraissait trop cruelle ou fausse. Ainsi, elle pouvait mettre en parallèle, bien que brièvement, la petite sensation qui donne lieu à l'émotion et le sens qui porte à croire ${ }^{16} »$. Alors qu'au moment de ses premières lectures elle avait éprouvé un sentiment diffus de réciprocité, maintenant le monde de Laure Angstelle prend place en elle différemment. Voici une très belle page du roman :

Maintenant le monde de Laure Angstelle avait en elle la portée d'une musique tout en durée qui la laissait devant sa table de travail comme un bloc de concentration; les yeux astreints au moindre détail pendant qu'au loin les images les plus intimes vacillaient, Maude Laures s'adaptait à toutes les intrigues pouvant, état d'alerte, disposer de sa ferveur ... et de sa froideur. Car à l'improviste «tromper la langue » lui venait comme une réplique nécessaire afin que soit reconstituée "la fiction », le contour tremblé de ses effets. La froideur de Maude Laures était un incommensurable désert blanc sillonné d'éclairs mauves. Il fallait beaucoup de rapidité dans le processus pour que la froideur ne soit point sécheresse, pour que Maude Laures abrite cet immense espace à découvert, recouvre chaque mot d'un autre mot sans que le premier ne sombre dans l'oubli ${ }^{17}$. 
Voilà la traduction - celle qui, disait Jacques Derrida, garde la mémoire endeuillée et endettée du corps premier qu'elle élève, sauve et relève ${ }^{18}$. Et justement, il est en permanence question de corps dans ce roman : les mots sont décrits comme des corps solides ${ }^{19}$, Maude se lance dans un corps à corps avec le livre, et la traduction devient vite une opération charnelle, une lutte ou une union avec la matière. Le moi devient par cette opération "substance mouvante » et la traductrice se glisse "anonyme et entière entre les pages ${ }^{20} »$; elle s'y glisse tout entière, car elle est là, elle est toujours présente. Et voilà que «tromper la langue» devient nécessaire pour reconstituer la fiction. La page citée ci-dessus précède immédiatement les notes rédigées par la traductrice sur les lieux, les objets et les personnages du récit, ainsi que des scènes qu'elle aura imaginé avoir lieu entre les personnages. C'est qu'afin d'obtenir la meilleure précision possible, Maude doit imaginer ce que le texte original ne révèle pas. Tout ce métarécit que Maude construit autour des lieux, des objets, des personnages et des dimensions, lui sert à remplir les zones d'ombre, à préciser le sens d'un récit premier qui reste énigmatique, insaisissable, elliptique. C'est qu'en traduisant, Maude ne cherche point à transporter de la manière la plus intacte un certain signifié dans un signifiant véhiculaire ; tout au contraire, elle cherche à éclairer le non-dit, à se dévoiler en dévoilant à elle-même ce qui est à l'origine de sa fascination, de son désir et de son trouble.

\section{La traduction}

On commence donc à entrevoir l'enjeu et le sens de cette traduction du français au français. On pourrait dire en termes benjaminiens qu'elle sert à éluder, voire à élider, la dimension de la communicabilité ${ }^{11}$, pour laisser place à ce qui reste, à ce qui a été depuis le début (depuis le Cratyle de Platon, si l'on veut) destiné à rester hors de toute réflexion théorique sur la langue ${ }^{22}$. Mais que reste-t-il ? Certainement pas ce que pense Walter Benjamin, du moins telle n'est pas la perspective partagée par Nicole Brossard. Non seulement la traduction du français au français élimine la question du «sens référentiel ", mais aussi elle met hors-jeu la question des langues comme systèmes de signes. Nous ne sommes pas dans l'horizon de la langue comme fragment d'une totalité adamique de rapports possibles à la réalité. Pour Walter Benjamin, la langue de la traduction fait allusion de manière embryonnaire et sous le mode de l'anticipation à ce que l'auteur appelle die reine Sprache, la pure langue ${ }^{23}$. Ce que Benjamin entend par pure langue n'est pas une sorte de logos sous-jacent à toutes les langues, mais à la fois la langue perdue (celle d'avant la confusion des langues et leurs devenirs multiples, c'està-dire la langue de l'origine adamique), et la langue-qui-vient, une langue plus grande faite de la réunion totalisante de tous les fragments que sont les langues naturelles; une langue qui, par conséquent, n'est envisageable qu'à partir d'un horizon messianique d'attente. Cette relation de toute langue naturelle avec la pure langue comme fragment d'une totalité à la fois originaire et à venir, fait en sorte que «la traduction sert donc en définitive la finalité de l'expression de la relation la plus intime des langues entre elles ${ }^{24} »$.

$\mathrm{Si}$, chez Walter Benjamin, il est question d'intimité entre les langues, chez Nicole Brossard l'intimité concerne les corps - des personnes aussi bien que des mots. En traduisant du français au français, on ne traduit pas la langue, on traduit le geste, l'intention qui donne origine à une certaine lexis pour autant qu'on puisse la saisir, 
c'est-à-dire reconstruire, ou mieux encore imaginer à partir des fulgurances de sens produites par notre acte de lecture. Afin de traduire, écrit Nicole Brossard dans son ouvrage consacré à la traduction, il faut non seulement accueillir le texte, mais «en assurer la reproduction fictive, fût-elle imaginaire, utopique, mentale ou plausible, dans une autre langue ${ }^{25}$ ». Car le processus du traduire relève à son tour d'un geste et d'une intention originaires, puisqu'il s'agit d'un acte de création, où il est encore question d'écriture, de venue à la parole. Et c'est dans le mystère de cette venue à la parole que se cache un certain non-dit... un non-dit qui serait le résultat - ou le résidu du passage d'une certaine urgence, vécue sous le mode de l'appel, à une réalisation langagière qui en serait la réponse.

Maintenant, on peut voir aisément comment le rapport au non-dit auquel je fais référence est intrinsèquement lié à la question posée par le roman de Nicole Brossard : qui devient qui en traduisant, en étant traduit? La langue, dit Nicole Brossard, est le théâtre de l'autre en soi. «J'ai mis de la distance entre ma langue maternelle et la réalité", écrit-elle dans un autre roman, intitulé La Capture $d u$ sombre ${ }^{26}$. Cette "hésitation identitielle", cette oscillation du soi, concerne autant ce que l'auteure appelle la partie invisible en nous que les liens qu'il faut établir entre l'invisible, l'indicible et notre rapport à la langue. Car la langue, écrit Nicole Brossard, "peut nous faire dire ce que nous ne croyons pas avoir pensé27 ${ }^{7}$. Ce n'est pas l'impensable, justement ; mais l'impensé. Qu'est-ce que pourrait être cette im-pensée ? Une impensée comme tout ce qui est de l'ordre du virtuel, à savoir les innombrables possibilités d'émerveillement dans la vie; mais aussi l'impensée comme ce que l'on ne pense pas avoir pensé. Si l'on ne pense pas avoir pensé quelque chose, cela signifie, d'une certaine façon, qu'on l'a quand même pensé, ou mieux qu'on l'a pensé autrement. Penser cette impensée qui n'est plus de l'ordre du virtuel et qui relève au contraire d'une véritable présence, quoique cachée, signifie en d'autres termes reconnaître une temporalité propre à la prise de conscience : le devenir conscient de quelque chose est moins une opération immédiate que le fruit d'un travail d'adéquation, d'équilibre, de dialectique entre l'ordre ou les ordres normatifs, ou si vous voulez la culture comme système de possibilités et d'interdits, et le désir - le désir comme instance de vie, facteur potentiel de trouble. Or, s'il y a un lien entre invisible, indicible et rapport à la langue, c'est parce que même notre langue peut se constituer - et se constitue - comme ordre normatif et lieu d'oppression. Voici, dans ce cadre, une citation qui n'aura guère besoin de commentaire :

Pourquoi suis-je si émue à propos de la langue comme ce jour où on m'a fait entendre le dvd de la troupe française de théâtre nommée Le Poème Harmonique? Cette troupe qui se consacre à reproduire le plus exactement possible les pièces de Molière telles qu'elles ont dû être jouées (éclairage, musique, mise en scène et surtout langue du xvII e siècle où l'on prononçait chaque syllabe, chaque $s$, et roulait tant que se pouvait les $r$ ). En quelques secondes, les ancêtres sont apparus. L'émotion s'est infiltrée, forte, entre ce que je connais de l'histoire québécoise et ce que je sais de la France et de sa littérature.

Émotions, oui, questions, bien évidemment. Que veut-on m'interdire en rendant ce $e$, indice du féminin, muet, cachant ainsi derrière le masculin des millions de femmes et conséquemment une partie de la réalité ? Pourquoi veut-on me faire croire qu'une cuisinière est « un fourneau de cuisine servant à chauffer et à cuire les aliments » et qu'une traductrice est « une machine à traduire et qu'elle peut être dite : traductrice de poche »? À quoi ressemblent le cœur et le contour de ma langue? Quel rôle son histoire joue-t-elle dans les peurs et les joies qui rôdent dans mes pensées ${ }^{28}$ ? 
17 La traduction - telle que nous avons essayé de la penser ici à partir du roman Le Désert mauve de Nicole Brossard - montre ainsi comme possibilité la plus propre la mise en langue de ce qui, dans le texte soi-disant original, n'a pas été dit, et qui seul peut affleurer dans la rencontre - toujours unique - entre un texte et cette occurrence concrète du vivant que l'on appelle lecteur ou lectrice. Telle serait au moins la tâche inévitable de tout traducteur et de toute traductrice.

\section{NOTES}

1. N. Brossard, Le Désert mauve, Montréal, Typo, coll. «Romans", 2010 (Première édition: Montréal, L'Hexagone, coll. « Fictions », 1987).

2. N. Brossard, Le Désert mauve, ouvr. cité, p. 33.

3. Ibid., p. 47.

4. Ibid., p. 53.

5. J'emploie le mot totale et ses dérivés pour faire signe à une clôture, un repliement sur le même qui ne voit pas - ou mieux, qui ne peut pas voir - ce que l'altérité pourrait lui apporter. C'est à cause de cette qualité totalisante que chaque locuteur vit dans l'illusion que sa propre langue peut couvrir la totalité du réel ; du moins, jusqu'à ce que l'apprentissage d'une autre langue ne le détrompe sur un tel fantôme.

6. Voir N. Brossard, Et me voici soudain en train de refaire le monde, Montréal, Mémoire d'encrier, coll. « Cadastres», 2015, p. 25.

7. Grazie - peut-être n'est-il pas inutile de le remarquer - s'écrit comme le mot «merci » en italien.

8. N. Brossard, Le Désert mauve, ouvr. cité, p. 55.

9. Ibid., p. 53.

10. Ibid., p. 75.

11. Dans Le Monolinguisme de l'autre (J. Derrida, Paris, Galilée, coll. « Incises », 1996), il est question du rapport, tout à fait singulier, à la langue française que pouvait avoir un Juif-Français-d'Algérie dans un moment historique précis, c'est-à-dire sous le régime vichyste. La singularité de cette condition permet toutefois au philosophe de saisir quelque chose de général du rapport à la langue maternelle - à toute langue maternelle, en tant qu'appartenance non-appartenante, en tant que langue de l'autre: «je n'ai qu'une langue, ce n'est pas la mienne », tel est le paradoxe que Derrida essaie de penser comme condition générale de l'être humain en tant qu'être-enlangue.

12. Voir A. Berman, L’Âge de la traduction: "La Tâche du traducteur» de Walter Benjamin, un commentaire, Saint Denis, Presses universitaires de Vincennes, coll. « Intempestives », 2008.

13. Ce que j'essaie de suggérer ici c'est que le premier récit par Laure Angstelle ne serait pas même pensable sans sa traduction, et qu'il ne peut prendre du sens qu'après sa réception et sa transformation par Maude Laures. Ceci n'est bien sûr qu'une intuition quelque peu imprudente : faudrait-il peut-être demander son avis à l'autrice?

14. N. Brossard, Le Désert mauve, ouvr. cité, p. 79.

15. Ibid., p. 86.

16. Ibid., p. 87.

17. Ibid., p. 88-89. 
18. Voir J. Derrida, Qu'est-ce qu'une traduction « relevante»?, Paris, L'Herne, coll. " Carnets de l'Herne », p. 71-72.

19. N. Brossard, Le Désert mauve, ouvr. cité, p. 201.

20. Ibid., p. 205.

21. Le célèbre essai de Walter Benjamin intitulé La Tâche du traducteur commence par écarter la perspective de la réception dans la considération de toute œuvre artistique. L'auteur se demande ce que dit une œuvre littéraire, ce qu'elle communique: «très peu pour qui la comprend. L'essentiel en elle n'est pas la communication ni l'énonciation ». Voir W. Benjamin, « La Tâche du traducteur ", dans Expérience et pauvreté ; suivi de Le Conteur ; La Tâche du traducteur, C. Cohen-Skalli (trad.), Paris, Payot, coll. « Petite Bibliothèque Payot », 2011, p. 110.

22. J'entends par là que dans la tradition philosophique occidentale l'approche dominante de la question du langage a toujours été orientée vers le rapport entre le mot et la chose, au détriment d'une exploration riche et détaillée du rapport, par exemple, entre la parole et le corps.

23. Voir W. Benjamin, « La Tâche du traducteur », ouvr. cité, p. 119-121.

24. Ibid., p. 116.

25. N. Brossard, Et me voici soudain..., ouvr. cité, p. 43.

26. N. Brossard, La Capture du sombre, Montréal, Leméac, 2007.

27. N. Brossard, Et me voici soudain..., ouvr. cité, p. 24.

28. Ibid., p. 26-27.

\section{RÉSUMÉS}

Et si au fond toute traduction n'était qu'une fiction? Voici l'hypothèse, autrement dit le point de départ, auquel nous conduisent la réflexion et la création - une création jamais disjointe de la réflexion - de la poète québécoise Nicole Brossard. L'auteure a en effet expérimenté dans sa fiction romanesque - en particulier avec le roman Le Désert mauve (1987) - une écriture comme traduction du français au français, d'une langue à la même langue. Cet article tâche de comprendre la signification d'une telle opération : s'agit-il d'un exercice simplement ludique ou bien ce roman nous offre-t-il une matière pour penser différemment la traduction et notre rapport à la langue?

In the 1987, the Québec poet Nicole Brossard published an astonishing novel called Le Désert Mauve (english trad. Mauve Desert), which fictionalises the translation process, conceived in the diegetic world as a translation from a language into the same language. Starting from an interpretation of Brossard's paradoxical example of translation, this article tries to raise the question of the real object of every translation process.

\section{AUTEUR}

\section{ANGELO VANNINI}

Écrivain et dramaturge, Angelo Vannini a enseigné la langue et la littérature italiennes à l'université de Paris-Nanterre et à l'université de Franche-Comté. Ses travaux de recherche portent sur le rapport entre philosophie et littérature, sur la philosophie de la traduction, ainsi 
que sur la réception des textes de l'antiquité gréco-romaine dans les littératures modernes et contemporaines. Il a récemment publié en Italie un livre intitulé L'intermissione dei cigni.

Cinquantanove giorni alla frontiera della letteratura (Arcipelago Itaca Edizioni, 2017), une méditation poétique sur la frontière comme lieu propre et propice à la littérature, en dialogue critique avec Platon, Ovide, Montaigne, Adorno, Celan, Derrida et Cixous. Ses pièces théâtrales ont été représentées à Milan (Triennale), à Paris (Centre Pompidou, Panthéon, École normale supérieure, mairie du $5^{\mathrm{e}}$ arrondissement) et à New York (théâtre La MaMa). 